\title{
Temporal Plasticity Involved in Recovery from Manual Dexterity Deficit after Motor Cortex Lesion in Macaque Monkeys
}

\author{
(D) Yumi Murata, ${ }^{1,2}$ Noriyuki Higo, ${ }^{1,3,4}$ Takuya Hayashi, ${ }^{5}$ Yukio Nishimura, ${ }^{4,6}$ Yoko Sugiyama, ${ }^{1,7}$ Takao Oishi, ${ }^{1,3,8}$ \\ Hideo Tsukada, ${ }^{3,9}$ Tadashi Isa, ${ }^{3,6}$ and Hirotaka Onoe ${ }^{3,5}$ \\ ${ }^{1}$ Human Technology Research Institute, National Institute of Advanced Industrial Science and Technology (AIST), Tsukuba, Ibaraki 3058568, Japan, \\ ${ }^{2}$ Research Fellow of the Japan Society for the Promotion of Science, Chiyoda-ku, Tokyo, 1020083, Japan, ${ }^{3}$ Core Research for Evolutional Science and \\ Technology, Japan Science and Technology Agency, Kawaguchi, Saitama, 3320012, Japan, 4 Precursory Research for Embryonic Science and Technology, \\ Japan Science and Technology Agency, Kawaguchi, Saitama, 3320012, Japan, ${ }^{5}$ Division of Bio-Function Dynamics Imaging, Center for Life Science \\ Technologies, RIKEN, Kobe, Hyogo, 6500047, Japan, ${ }^{6}$ Department of Developmental Physiology, National Institute for Physiological Sciences, National \\ Institutes of Natural Sciences, Okazaki, Aichi, 4448585, Japan, ${ }^{7}$ Graduate School of Comprehensive Human Science, University of Tsukuba, Tsukuba, \\ Ibaraki, 3058577, Japan, ${ }^{8}$ Department of Cellular and Molecular Biology, Primate Research Institute, Kyoto University, Inuyama, Aichi, 4848506, Japan, and \\ ${ }^{9}$ Central Research Laboratory, Hamamatsu Photonics K.K., Hamamatsu, Shizuoka, 4348601, Japan
}

The question of how intensive motor training restores motor function after brain damage or stroke remains unresolved. Here we show that the ipsilesional ventral premotor cortex (PMv) and perilesional primary motor cortex (M1) of rhesus macaque monkeys are involved in the recovery of manual dexterity after a lesion of M1. A focal lesion of the hand digit area in M1 was made by means of ibotenic acid injection. This lesion initially caused flaccid paralysis in the contralateral hand but was followed by functional recovery of hand movements, including precision grip, during the course of daily postlesion motor training. Brain imaging of regional cerebral blood flow by means of $\mathrm{H}_{2}{ }^{15} \mathrm{O}$-positron emission tomography revealed enhanced activity of the PMv during the early postrecovery period and increased functional connectivity within M1 during the late postrecovery period. The causal role of these areas in motor recovery was confirmed by means of pharmacological inactivation by muscimol during the different recovery periods. These findings indicate that, in both the remaining primary motor and premotor cortical areas, time-dependent plastic changes in neural activity and connectivity are involved in functional recovery from the motor deficit caused by the M1 lesion. Therefore, it is likely that the PMv, an area distant from the core of the lesion, plays an important role during the early postrecovery period, whereas the perilesional M1 contributes to functional recovery especially during the late postrecovery period.

Key words: brain activation; functional compensation; macaque monkey; precision grip; primate

\section{Introduction}

Rehabilitative training can promote recovery of motor deficits in stroke patients, but the mechanisms underlying the recovery are not fully understood. This is partly due to the difficulty in controlling for the variability of stroke lesions and the disabilities

\footnotetext{
Received April 17, 2014; revised 0ct. 9, 2014; accepted 0ct. 31, 2014

Author contributions: Y.M., N.H., Y.N., T.O., T.I., and H.O. designed research; Y.M., N.H., T.H., Y.N., Y.S., and H.O. performed research; H.T. contributed unpublished reagents/analytic tools; Y.M., N.H., and T.H. analyzed data; Y.M. and N.H. wrote the paper.

This work was supported by a Japan Society for the Promotion of Science Research Fellowship for Young Scientists, as well as the Precursory Research for Embryonic Science and Technology and Core Research for Evolutional Science and Technology programs of the Japan Science and Technology Agency. We thank Dr. I. Takashima, Dr. T. Kakiuchi, Dr. H. Oba, M. Kawahara, K. Onoe, and A. Muramatsu for excellent technical assistance; and T. Takasu for superb care of the animals.

The authors declare no competing financial interests.

This article is freely available online through the J Neurosci Author Open Choice option.

Correspondence should be addressed to Dr. Yumi Murata, Systems Neuroscience Group, Human Technology Research Institute, National Institute of Advanced Industrial Science and Technology, Tsukuba Central 2, 1-1-1 Umezono, Tsukuba, Ibaraki 3058568, Japan. E-mail: murata.ym@aist.go.jp.

DOI:10.1523/JNEUROSCI.1737-14.2015

Copyright $\odot 2015$ the authors $\quad 0270-6474 / 15 / 350084-12 \$ 15.00 / 0$
}

they cause. Experimental animal studies, which allow for these factors to be controlled, provide information essential for understanding the underlying brain mechanisms of functional recovery from brain damage. In particular, in the field of motor recovery, lesioning of the primary motor cortex (M1) in nonhuman primates is a well-established experimental technique (Glees and Cole, 1950; Travis, 1955; Passingham et al., 1983; Nudo et al., 1996; Friel and Nudo, 1998; Liu and Rouiller, 1999; for review, see Vilensky and Gilman, 2002; Dancause et al., 2005).

Several studies have shown that functional and structural reorganization in a part of the undamaged brain may be involved in training-induced functional recovery after focal damage to M1 (Nudo et al., 1996; Frost et al., 2003; Dancause et al., 2005). These prior studies, however, assessed reorganization in only a limited part of the brain by using intracortical microstimulation (ICMS) under ketamine sedation or by performing anatomical analysis with a tracer injection in squirrel monkeys.

We recently reported that, when rhesus monkeys given a lesion of the M1 digit area were trained for a particular set of motor tasks, they exhibited gradual recovery of dexterous hand move- 
Table 1. M1-lesioned monkeys used in the present study

\begin{tabular}{lllll}
\hline Monkey & Experiment & Sex & Weight $(\mathrm{kg})$ & $\begin{array}{l}\text { Lesion volume } \\
\text { (\% whole digit area) }\end{array}$ \\
\hline Re & Brain imaging & Male & 4.8 & 69.0 \\
$\mathrm{Hw}$ & Brain imaging & Male & 4.6 & 83.5 \\
$\mathrm{Ja}$ & Pharmacological inactivation & Male & 7.9 & 78.0 \\
$\mathrm{Ki}$ & Pharmacological inactivation & Male & 6.8 & 67.5 \\
\hline
\end{tabular}

ments (Murata et al., 2008). We used rhesus macaques because, among nonhuman primate species, they are more comparable with humans with respect to the anatomical structures of the motor cortex and corticospinal tract than are other monkeys used in experimental research (e.g., squirrel monkeys and marmosets) (for review, see Courtine et al., 2007). This motor system homology with humans, in combination with the relatively large macaque brain, makes imaging data obtained in rhesus monkeys comparable with those examined in clinical research. Therefore, studying the rhesus monkey can facilitate the translation of findings to human patients.

The aim of the present study was to demonstrate functional reorganization of brain activity in M1-lesioned macaque monkeys and to relate this to the performance of dexterous hand movements, a motor function that is important in human healthrelated quality of life. We assessed whole-brain regional cerebral blood flow (rCBF) as an index of brain activity by using $\mathrm{H}_{2}{ }^{15} \mathrm{O}$ positron emission tomography (PET) scans during performance of precision grip in awake and behaving animals. To confirm that the areas identified by PET were causally related to the recovered behavior, we further performed a study in which we pharmacologically inactivated each region whose increase in $\mathrm{rCBF}$ was positively correlated with the recovered behavior by using muscimol, a $\mathrm{GABA}_{\mathrm{A}}$ receptor agonist. Thus, we confirmed whether local inactivation of the positive regions identified by PET imaging would redisable the recovered hand movements as assessed by the precision grip task.

\section{Materials and Methods}

Subjects. Four male rhesus monkeys (Macaca mulatta) were used in the PET imaging and inactivation studies (Table 1). Before the lesion, the monkeys underwent prelesion training that involved a small-object retrieval task, and topographic motor maps of M1 and the ventral premotor area (PMv) were constructed by using ICMS. Ibotenic acid was then injected to destroy the digit region of M1. Flaccid paralysis in the contralateral hand caused by the M1 lesion functionally recovered during 1 month of daily postlesion motor training (see Fig. $1 A, B$ ). A series of PET scans was conducted in two monkeys (Re and $\mathrm{Hw}$ ). Two time periods were selected to investigate the time-dependent compensatory mechanisms involved. Flaccid hand paralysis occurred after ibotenic acid lesion of M1, and dexterous hand movements, including precision grip, became considerably restored during the first month after M1 lesion (see Fig. $1 A$ ). The period 1-2 months after lesion, which is when the mean success rate was below the $95 \%$ confidence level for prelesion performance, was selected as the period just after recovery (i.e., the early postrecovery period) (see Fig. 1B). The period 3-4 months after lesion, which is when $>1$ month had passed after the mean success rate increased to within the $95 \%$ confidence level, was selected as the plateau phase of recovery (i.e., the late postrecovery period). We did not perform PET imaging during the very early recovery period ( $<1$ month after lesion) because behavioral performance during this period was so severely impaired that behavior-related activities were unlikely to be detected at sufficient resolution by the current PET system. Thus, the earliest PET study was performed when the behavior level was almost restored (1-2 months after lesion) so that behavior-related brain activity could be robustly assessed. Furthermore, in two other monkeys (Ja and Ki), a pharmacological inactivation study with muscimol was performed in three exper- imental periods identical to those in which PET imaging was performed to confirm that the areas identified by PET were causally related to the recovered behavior. In addition, one male monkey was used to determine the extent of muscimol spread. No statistical methods were used to predetermine sample sizes. We attempted to minimize the number of monkeys used on the basis of ethical considerations and data similarity; our sample sizes are similar to those reported in previous publications by our group and others. The monkeys were $\geq 4$ years of age and purchased from a local provider. The animal use protocol was approved by the Institutional Animal Care and Use Committee of the National Institutes of Natural Sciences and the National Institute of Advanced Industrial Science and Technology, Japan. The monkeys were housed in adjoining individual primate cages (width, $750 \mathrm{~mm}$; length, $950 \mathrm{~mm}$; height, 930 $\mathrm{mm}$ ) allowing social interactions under controlled conditions of humidity, temperature, and light; they were monitored daily by the researchers and animal care staff to ensure their health and welfare. The housing area was maintained on a $12 \mathrm{~h}$ light- $12 \mathrm{~h}$ dark cycle, and all of the experiments were conducted during the light cycle. Environmental enrichment consisted of commercial toys. A commercial primate diet and fresh fruit and vegetables were provided daily, and water was provided in a drinking bottle and freshened daily. We used naive monkeys without any history of experimentation. Adequate measures were taken to minimize pain or discomfort in accordance with the National Institutes of Health Guidelines for the Care and Use of Laboratory Animals.

Lesion induction. An artificial lesion was induced in the hand digit area of M1 by using the same procedure as that used in our previous study (Murata et al., 2008). Topographic motor maps of M1 and PMv were constructed by using ICMS. Under sterile conditions and pentobarbital anesthesia $(25 \mathrm{mg} / \mathrm{kg})$, a craniotomy was made over M1 and PMv, and a stainless steel chamber and head holders were then affixed to the skull with dental acrylic. ICMS was conducted under ketamine anesthesia, with the rate of ketamine infusion adjusted to maintain relatively stable anesthesia. A flexible tungsten microelectrode (MicroProbe) was advanced perpendicular to the cortical surface to a depth of 5-15 $\mathrm{mm}$ at intervals of $500 \mu \mathrm{m}$ by using a hydraulic microdrive. A conventional ICMS technique (1-100 $\mu \mathrm{A}, 200 \mu \mathrm{s}$ in duration, 12 pulses, monophasic cathodal pulses at $333 \mathrm{~Hz}$ ) was used to evoke movement at each electrode penetration site. Electrophysiological recording was also conducted to determine the border between the primary motor and sensory areas by using a head amplifier (JH-110J, Nihon Kohden) and main amplifier (MEG-5100, Nihon Kohden); the sensory response was investigated by applying light tactile stimuli to the face, digit, wrist, and forearm of the monkey with a small hand-held brush. We defined the digit region of M1 as a $2 \mathrm{~mm}$ square of cortical surface centered on the penetration site that satisfied at least one of the following criteria: (1) digit movements were obtained at a current lower than $10 \mu \mathrm{A}$, or (2) digit movements were obtained while the movements of the other body parts were not obtained with the lowest threshold current, and the current was $<20 \mu \mathrm{A}$. Ibotenic acid $(15 \mu \mathrm{g} / \mu \mathrm{l}$ in $0.1 \mathrm{M}$ PBS, pH 7.4; 7.0-9.9 $\mu \mathrm{l})$ was then injected intracortically to destroy the digit region of M1 contralateral to the preferred hand. Multiple injections were used to spread ibotenic acid throughout the digit region, with $0.8-1.0 \mu \mathrm{l}$ injected at a rate of $0.2 \mu \mathrm{l}$ per minute at each injection site (8-10 injection sites for each monkey). Daily hand performance before and after lesion was evaluated by means of a smallobject retrieval task.

Determination of lesion volume. Lesion locations were also determined by using the same procedure we used in our previous study (Murata et al., 2008). Briefly, animals were perfused through the ascending aorta with $0.5 \mathrm{~L}$ of ice-cold saline containing $2 \mathrm{ml}(2000 \mathrm{U})$ of heparin sodium followed by ice-cold fixative containing $4 \%$ PFA and $0.1 \%$ glutaraldehyde. Brain segments were sectioned coronally at a thickness of $16 \mu \mathrm{m}$ on a cryostat (Cryocut 3000, Leica) and then Nissl-stained with cresyl fast violet. Images of the Nissl-stained sections were photographed under an Olympus BX60 microscope by using a 3CCD color video camera (DXC950, Sony) and then digitized with an image analysis system (MCID, Imaging Research). The lesioned area was defined as the area of gliosis as shown by Nissl staining, and unbiased volumes of the lesioned region and 
A

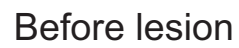

2 days

1

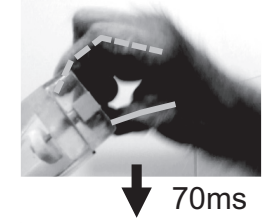

2

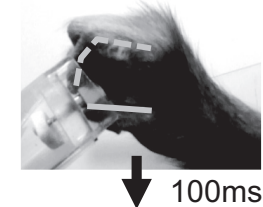

3

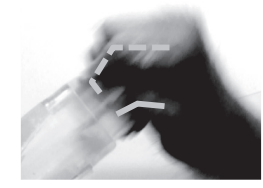

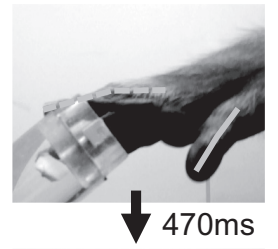
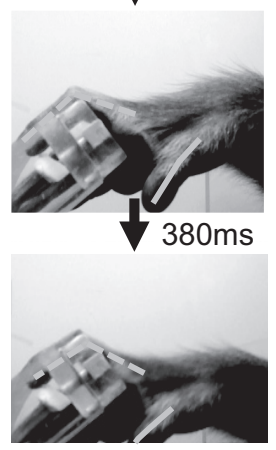

29 days after lesion
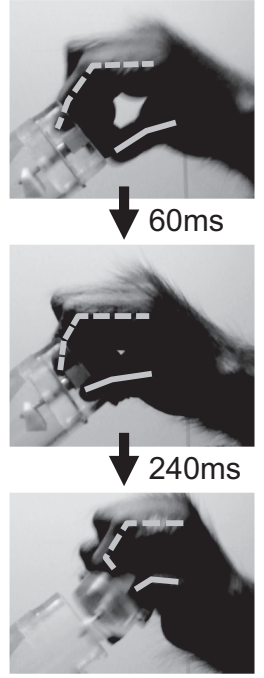

B

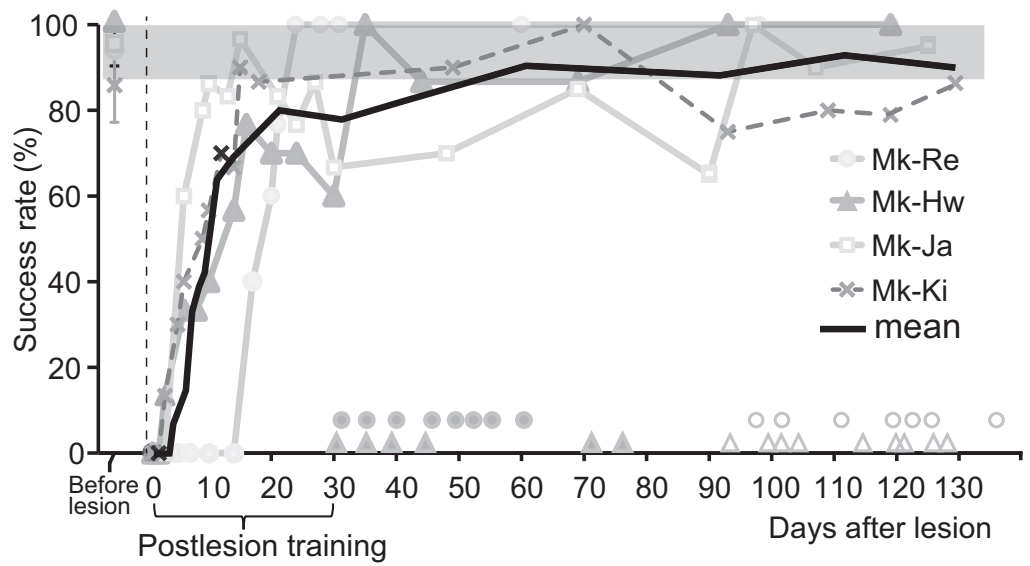

C

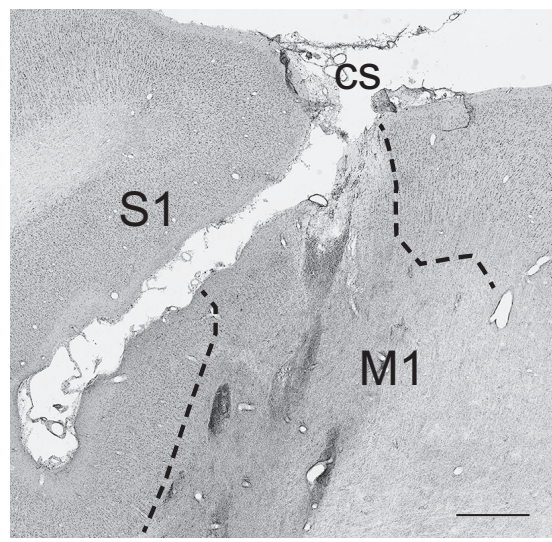

D

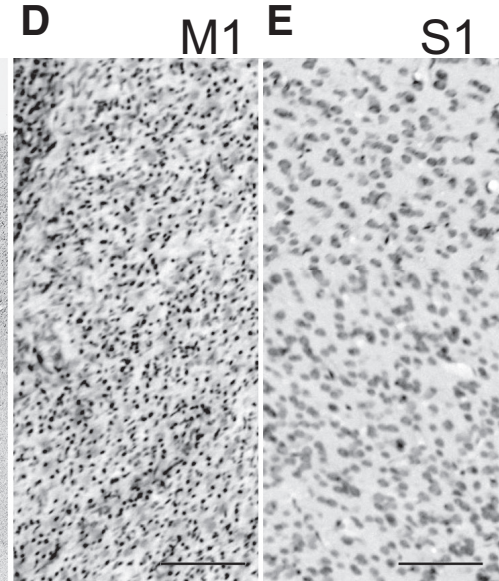

Figure 1. Recovery of precision grip after lesion of M1. A, Sequence of photographs showing hand and digit movements while performing the precision grip task (Monkey [Mk]-Ja), in which monkeys grasped a small cubic knob attached to a plastic disk and retrieved it from a tube through a slit aperture. The joints and tips of the thumb and index finger are linked by solid and dotted lines, respectively. Rows 1, 2, and 3 show the moment of contact of the tips of the index finger with the aperture of the slot, contact between the tip of the index finger and the square knob, and retrieval of the object, respectively. $\boldsymbol{B}$, Time course of changes in the success rate of precision grip in the monkeys used in the $\mathrm{H}_{2}{ }^{15} 0$-PET imaging (Mk-Re and Mk-Hw) and inactivation analyses (Mk-Ja and Mk-Ki). A successful trial was defined as the removal of the object by using the precision grip between the tip of the index finger and thumb. Shaded gray region represents the $95 \%$ confidence interval for prelesion performance. Filled circles and triangles above the $x$-axis represent the days on which PET scans during the early postrecovery period were performed for Mk-Re and the M1 digit region were calculated based on Cavalieri's principle (Mayhew, 1992).

Behavioral tests. Performance of hand movements was evaluated daily by means of a smallobject retrieval task closely resembling that used in our previous studies (Nishimura et al., 2007; Murata et al., 2008; Higo et al., 2009). In this task, the monkeys either retrieve a small piece of sweet potato $(7 \times 7 \times 7 \mathrm{~mm}$ in size $)$ through a narrow vertical slit $(10 \mathrm{~mm}$ in width), or grasp a small knob $(7 \times 7 \times 7 \mathrm{~mm}$ in size) on a disk ( $20 \mathrm{~mm}$ in diameter) attached to a piece of sweet potato, which they retrieve through a narrow vertical slit (10 $\mathrm{mm}$ in width) by using both the index finger and thumb. The test session to evaluate hand performance consisted of 20 trials. In addition, the monkeys underwent daily postlesion training after the M1 lesion for $>30 \mathrm{~min}$ a day, 5 days a week, by using the retrieval task. Moreover, during the postlesion training period (from day 1 to $\sim 1$ month, days 28-35, after the M1 lesion), the monkeys wore a jacket (Alice King Chatham Medical Arts, or Lomir Biomedical) with a dead-end sleeve covering the unaffected hand, forcing them to use the affected hand, as in our previous studies (Murata et al., 2008; Sugiyama et al., 2013). This method resembles that used in constraint-induced movement therapy, which has been widely adopted in the rehabilitation of stroke patients (Taub et al., 2002). Grip movement was recorded by using a CCD camera (XC-HR50, Sony) and Windows Live Movie Maker 2011 (Microsoft) was used for video analysis.

PET scanning. PET scanning was performed as described previously (Nishimura et al., 2007) with some modifications. A series of PET scans was conducted during the prelesion, early postrecovery (1-2 months after the M1 lesion), and late postrecovery periods (3-4 months after the M1 lesion). The monkeys were intensively trained, both outside and inside the PET scanner, to be seated on a monkey chair and perform the small-object retrieval task at a constant pace (once every $5 \mathrm{~s}$ ). In addition, they were trained on a control task, in which the food piece was stuck on the tip of a rod and given to their mouth through a long tube with the same frequency and trials as in the precision grip task but with both arms restricted.

$\mathrm{H}_{2}{ }^{15} \mathrm{O}$-PET scans were performed with a 3D mode acquisition by using a high-resolution animal PET scanner (SHR-7700, Hamamatsu

Mk-Hw, respectively. Open circles and triangles represent the days on which PET scans during the late postrecovery period were performed for the corresponding animals. $C-E$, A Nissstained section of Mk-Ki showing a representative lesion of M1. Ibotenic acid injection in M1 resulted in loss of neurons and gliosis $(\boldsymbol{D})$, whereas the neighboring primary sensory area $(\mathrm{S} 1)$ remained intact $(\boldsymbol{E})$. Dotted lines indicate the boundaries between the normal and lesioned areas. We defined the lesioned area as the area of gliosis, as in our previous study ( $\mathrm{Mu}$ rata et al., 2008). CS, Central sulcus. Scale bars: $\boldsymbol{C}, 1 \mathrm{~mm} ; \boldsymbol{D}, \boldsymbol{E}$, $100 \mu \mathrm{m}$. 


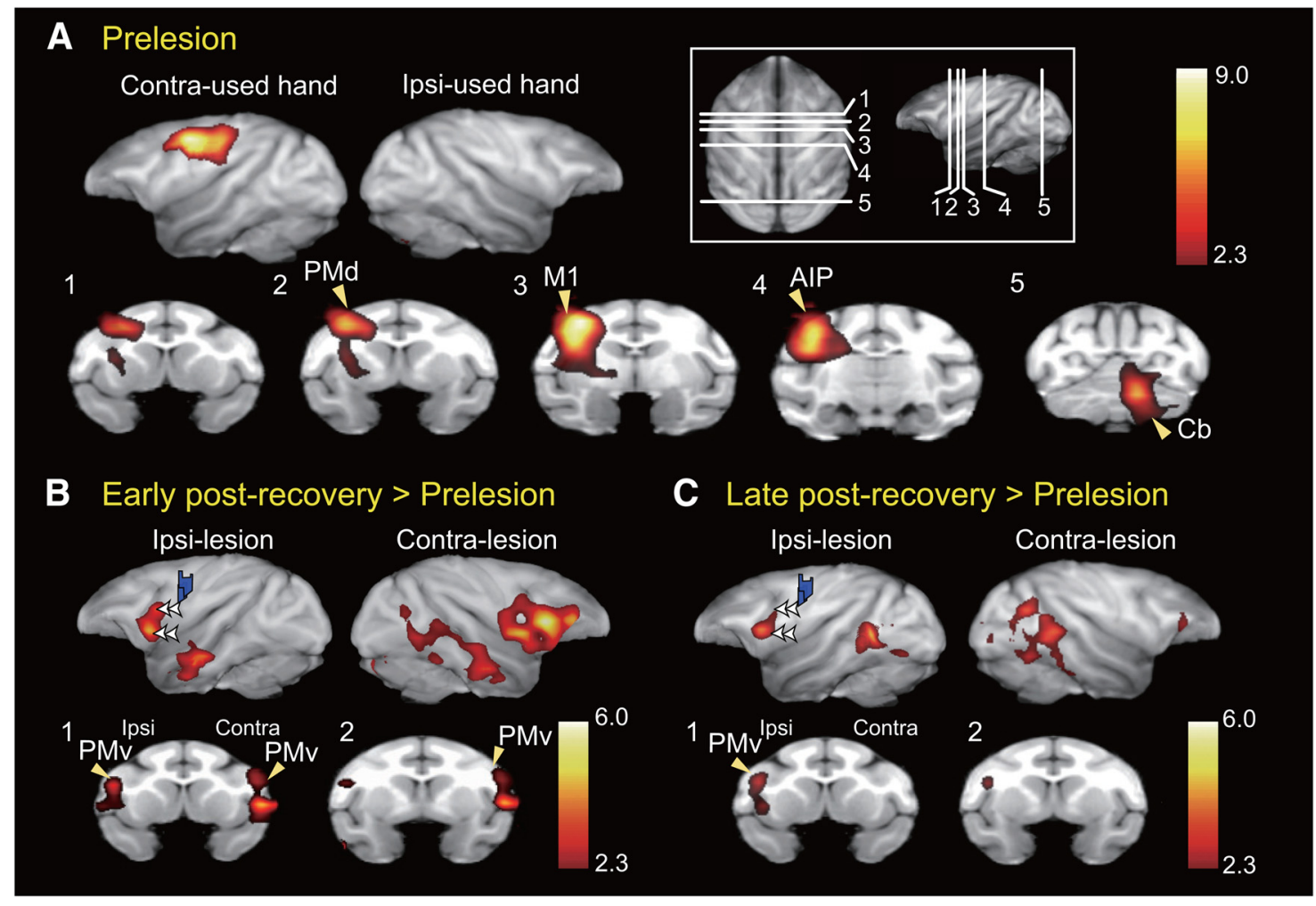

Figure 2. Increased brain activation during functional recovery. $A$, Brain areas activated during the precision grip task before the lesion were quantified in terms of increased $\mathrm{rCBF}$ by using $\mathrm{H}_{2}{ }^{15}$ - -PET. The rCBF during the control task was subtracted from that during the precision grip task. Brain areas with significantly increased $\mathrm{rCBF}$ ( $p<0.05$, corrected for multiple comparisons in two monkeys) are superimposed on a macaque monkey brain MRI template. The significance level is given in terms of a $Z$ score represented on a color scale. Top row, Views from both hemispheres contralateral and ipsilateral to the hand used in the precision grip task. Bottom row, Coronal sections at the level of lines shown in the inset. AIP, Anterior intraparietal cortex; $\mathrm{Cb}$, cerebellum; PMd, dorsal premotor cortex. $\boldsymbol{B}, \boldsymbol{C}$, Activation during early $(\boldsymbol{B})$ and late $(\boldsymbol{C})$ postrecovery periods ( $\mathrm{r}(\mathrm{BF}$ during the precision grip task $-\mathrm{r}(\mathrm{BF}$ during the control task) was compared with that during the prelesion stage. Blue areas represent histologically confirmed lesions. $B, C$, Double arrowheads in the top row indicate the same subregions of the PMv; these subregions showed increased activation during the early but not late postrecovery periods.

Table 2. Statistical analysis of the increase in rCBF in preoperative, early postrecovery, and late postrecovery periods ${ }^{a}$

\begin{tabular}{llcccc}
\hline Area & Side & Zscore & $x$ & $y$ & $z(\mathrm{~mm})$ \\
\hline Preoperative stage (precision grip & - control) & & & \\
M1 & Contra-used hand & 9.11 & 16.5 & 18.5 & 30.5 \\
Cb & Ipsi-used hand & 6.7 & -6 & -14.5 & 6 \\
Area 5 & Contra-used hand & 4.23 & 14 & 4.5 & 30 \\
S1 & Contra-used hand & 4.14 & 24.5 & 9.5 & 34 \\
PMd & Contra-used hand & 5.23 & 12 & 25 & 29 \\
Early postrecovery (precision grip - control) $>$ prelesion (precision grip - control) & \\
PMv & Contra & 5.80 & -23.5 & 30.5 & 18.5 \\
PMv & Ipsi & 4.67 & 25 & 28 & 15 \\
TE & Ipsi & 4.17 & 29 & -7 & -8 \\
Cb & Contra & 3.65 & -14.5 & -16 & 6 \\
S2 & Contra & 4.71 & -27 & 23 & 15 \\
Late postrecovery (precision grip - control) $>$ prelesion (precision grip - control) & \\
PMv & Ipsi & 4.29 & 24 & 29 & 18 \\
V4/TE0 & Contra & 4.11 & -29 & -2 & 16 \\
V4/TE0 & Ipsi & 5.6 & 25 & -4 & 17 \\
\hline
\end{tabular}

The rCBF was related to functional recovery during both the early and late postrecovery periods compared with that during the prelesion stage. $Z$ scores of the peak voxels are indicated. The locations are indicated by the positions along the $x$ (ipsilateral-contralateral), $y$ (rostrocaudal), and $z$ (dorsoventral) axes of the Horsley-Clarke stereotaxic coordinates. Cb, Cerebellar cortex; contra, contralesional hemisphere; ipsi, ipsilesional hemisphere; PMd, dorsal premotor area; S2, secondary somatosensory area; TEO, area TE0; V4, fourth visual area.

Photonics). The transaxial resolution of the PET scanner was $2.6 \mathrm{~mm}$ at full-width half-maximum. The monkey was seated on a monkey chair in the PET scanner without anesthesia. PET scans were performed in an upright sitting position in a scanner that was tilted at $80^{\circ}$ from the vertical position to expand the view of the monkey. A 30 min transmission scan was obtained with a rotating ${ }^{68} \mathrm{Ge}-{ }^{68} \mathrm{Ga}$ pin source to evaluate the relative attenuation factor for image reconstruction. After the delivery of a bolus of $\mathrm{H}_{2}{ }^{15} \mathrm{O}(\sim 300 \mathrm{MBq}$ in $1.5 \mathrm{ml}$ followed by $1.0 \mathrm{ml}$ of saline) via a cannula placed into the sural vein, the scan was initiated automatically when the radioactivity in the brain was $>30 \mathrm{kcps}$. The monkey was allowed to begin the behavioral task ( 20 trials) $\sim 10 \mathrm{~s}$ before the start of the PET scan. During the scan, the monkeys performed a series of reachgrip-retrieve-eat movements consisting of 20 trials, each of which was initiated with $5 \mathrm{~s}$ intervals. In addition, brain activation during the control task was also recorded in separate sessions randomly mixed with sessions of the precision grip task. PET data were collected for $80 \mathrm{~s} \mathrm{(one}$ $40 \mathrm{~s}$ frame followed by four $10 \mathrm{~s}$ frames). During the scanning session, the movements of the monkey were continuously video-recorded. If the monkey did not start from the fixed starting point, or did not start reaching immediately (within $<15$ frames $=500 \mathrm{~ms}$ ) after presentation of the food, or did not move the hand directly to the food piece, the data from the session were excluded from the analysis. Approximately 20 PET scans were performed per day with an interscan interval of $\sim 15 \mathrm{~min}$. PET experiments were conducted twice a week. The PET data within each experimental period, prelesion (47 scans for Mk-Re, and 55 scans for $\mathrm{Mk}-\mathrm{Hw}$ ), early postrecovery (47 scans for Mk-Re, and 47 scans for Mk$\mathrm{Hw}$ ), and late postrecovery periods ( 51 scans for Mk-Re, and 54 scans for $\mathrm{Mk}-\mathrm{Hw}$ ) were merged and then processed as detailed previously (Nishimura et al., 2007; Hayashi et al., 2013) with some modifications. In addition, ${ }^{18} \mathrm{~F}$-2-fluoro-2-deoxy-D-glucose (FDG) PET scan was performed for each subject by injecting a bolus of ${ }^{18} \mathrm{~F}-\mathrm{FDG}(40 \mathrm{MBq} / \mathrm{kg})$ to obtain spatial information on the brain for masking nonbrain structures of the $\mathrm{H}_{2}{ }^{15} \mathrm{O}$-PET images.

For registration of PET data, high-resolution whole-brain MRI images were obtained by using a conventional T1-weighted, fast-spin echo sequence (in-plane resolution, $0.80 \times 0.80 \mathrm{~mm}$; slice thickness, $0.81 \mathrm{~mm}$ ) 
with a 3.0 Tesla MR scanner. Whole-brain MRI images were obtained during the prelesion and postlesion periods to which the PET data obtained during the corresponding two periods were coregistered and spatially normalized. We also confirmed that distortion of the brain structure induced by the ibotenic acid lesion was negligible.

Analysis of PET scan data. The PET data were processed as described previously (Nishimura et al., 2007; Hayashi et al., 2013) with some modifications. PET images obtained from scan sessions that satisfied the behavioral criteria were summated for their first $60 \mathrm{~s}$ epochs (including task performance of 12 trials) and were used for statistical analysis. The reconstruction was performed on projection data, after which images were corrected for attenuation by using a transmission scan with a $4.0 \mathrm{~mm}$ Hanning filter. The voxel size in the reconstructed brain images was $1.2 \times 1.2 \times 3.6 \mathrm{~mm}$. Forty scans were conducted in both tasks for every stage. PET data were processed by using FMRI Expert Analysis Tool Version 5.98, which is part of the FMRIB Software Library (www.fmrib.ox.ac. $\mathrm{uk} / \mathrm{fs} \mathrm{l})$. We first corrected the data of each animal for motion by using the FMRIB Linear Image Registration Tool (MCFLIRT) (Jenkinson et al., 2002). We performed independent component analyses using Multivariate Exploratory Linear Optimized Decomposition into Independent Components (MELODIC)

to remove noise components, such as radioactivity accumulation in the temporal and posterior neck muscles, because either may affect intrabrain radioactivity by the partial volume effect. Nonbrain structures were removed by using the Brain Extraction Tool (Smith, 2002). The data were merged into $4 \mathrm{D}$ data at each stage for each animal.

Subsequently, first-level statistical analysis was performed on the 4D data by using the GLM to identify brain areas that showed higher rCBF signals during the precision grip task. The design matrix modeled regressors for the block of the precision grip task and control task along with a regressor for the global values of $\mathrm{CBF}$ for each volume. The contrast of interest was precision grip task $>$ control task, and the resulting statistical map was spatially normalized to study standard macaque space by using transformation matrices determined from structural MRI data. These contrasts were then applied to higher-level fixed-effect analyses in each animal for the average effect of prelesion, as well as for the effect of stage (preoperative stage $<$ early postrecovery period or preoperative stage $<$ late postrecovery period), to detect the functionally enhanced activity that may be related to recovery of the precision task. This approach was adopted previously in human studies and found to be causal for functional compensation by using a sequential virtual-lesioning technique (Lee et al., 2003). We then combined each contrast across subjects for testing mixed effects. Statistical analyses were performed by using the FMRIB Improved Linear Model (Woolrich et al., 2001), and Z (Gaussianized T/F) statistic images were thresholded by using clusters determined by $Z>2.3$ and a corrected cluster significance threshold of $p=$ 0.05 (Worsley et al., 1992).

Next, psychophysiological interaction (PPI) analysis was performed to localize changes in activity correlated with that of the M1 hand area, including the lesion site. This approach, which looks for functional connectivity seeding from a dysfunctional area, was motivated by previous work demonstrating potential compensatory areas after virtual lesioning in the human brain (Lee et al., 2003; O'Shea et al., 2007; Hayashi et al., 2013). Therefore, an ROI was placed in the core of the M1 hand area $(x=$ 18.3, $y=15.7, z=27.1,249$ voxels, $31.1 \mathrm{~mm}^{3}$ ) determined based on a functional ICMS map of the hand area (see Fig. $5 \mathrm{~A}$ ). This area was colocalized with the task-induced activation in the preoperative stage and also confirmed to lose a certain percentage of neurons as described in
Ipsi- Contra-lesion

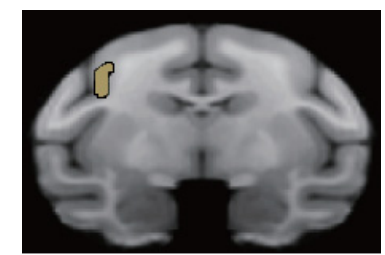

E

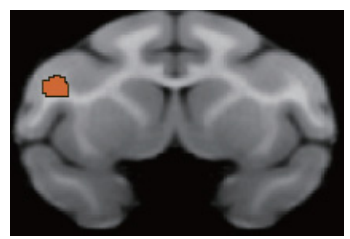

$\mathbf{F}$

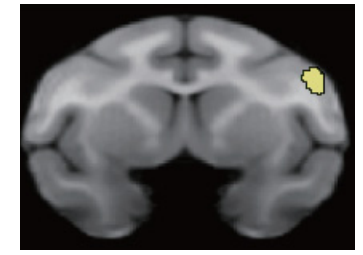

Results. The first-level design matrix for the PPI analysis included three regressors: those for the main effects of the precision grip task $(\mathrm{P})$ and activity in lesioned M1 (L), as well as one for the psychophysiological interaction (PPI-P) comprising an interaction term between $\mathrm{L}$ and $\mathrm{P}$. Additional regressors for the block of the control task (C), its PPI (PPI-C, the interaction between $\mathrm{L}$ and $\mathrm{C}$ ), and estimated motion parameters were also entered as confounds. Our contrast of interest was PPI-P (i.e., the interaction between the effect of precision grip task and activity of the M1 hand area, including the lesion site). The results of the first-level PPI were further fed into a higher-level within-subject fixed-effect analysis for the factorial effects of M1 lesion on the first-level contrasts and then into a random-effects analysis across subjects. We report peaks meeting a threshold of $Z>2.3$ ( $p<0.01$, uncorrected for multiple comparisons, one-tailed) and a cluster extent of 30 voxels. A spherical ROI in the perilesional M1 centered on peaks of clusters was thus identified. The mean \%rCBF signal change in the ROI was calculated for the precision grip task. These values were plotted against the equivalent values for the M1 hand area, including the lesion site (seed region), and regression lines were fitted to the data prelesion and during the two postrecovery periods for the purpose of visualizing the PPI effects.

Inactivation study. The topographic maps constructed before the lesion were used to determine the injection sites. Unit recordings and ICMS were performed with a microelectrode at the site chosen for the injection to confirm the depth in the gray matter. The microelectrode was then withdrawn and replaced by a stainless steel microsyringe (MS05 , Ito). Muscimol, a GABA receptor agonist (Sigma; $5 \mu \mathrm{g} / \mu \mathrm{l}$, dissolved in $0.1 \mathrm{M}$ phosphate buffer at $\mathrm{pH} 7.4$ ), was slowly injected with pressure at a rate of $0.2 \mu \mathrm{l}$ per minute by using an automatic microinjector (IMS-10, Narishige). Although we performed systematic ICMS mapping during the postrecovery periods after lesioning M1, the threshold for inducing body movements increased in many penetrations in the perilesional area, and ICMS up to $100 \mu \mathrm{A}$ did not elicit any hand movement in the ipsilesional PMv, as we have reported in a recent study (Higo et al., 2013). Accordingly, we decided to inject muscimol into the center of the digit area at the preoperative stage using the same coordinate system for the hand digit area of the ipsilesional M1 (M1-h) and the hand digit area of $\mathrm{PMv}(\mathrm{PMv}-\mathrm{h})$. Muscimol solution $(3 \mu \mathrm{l})$ was injected into a single injec- 


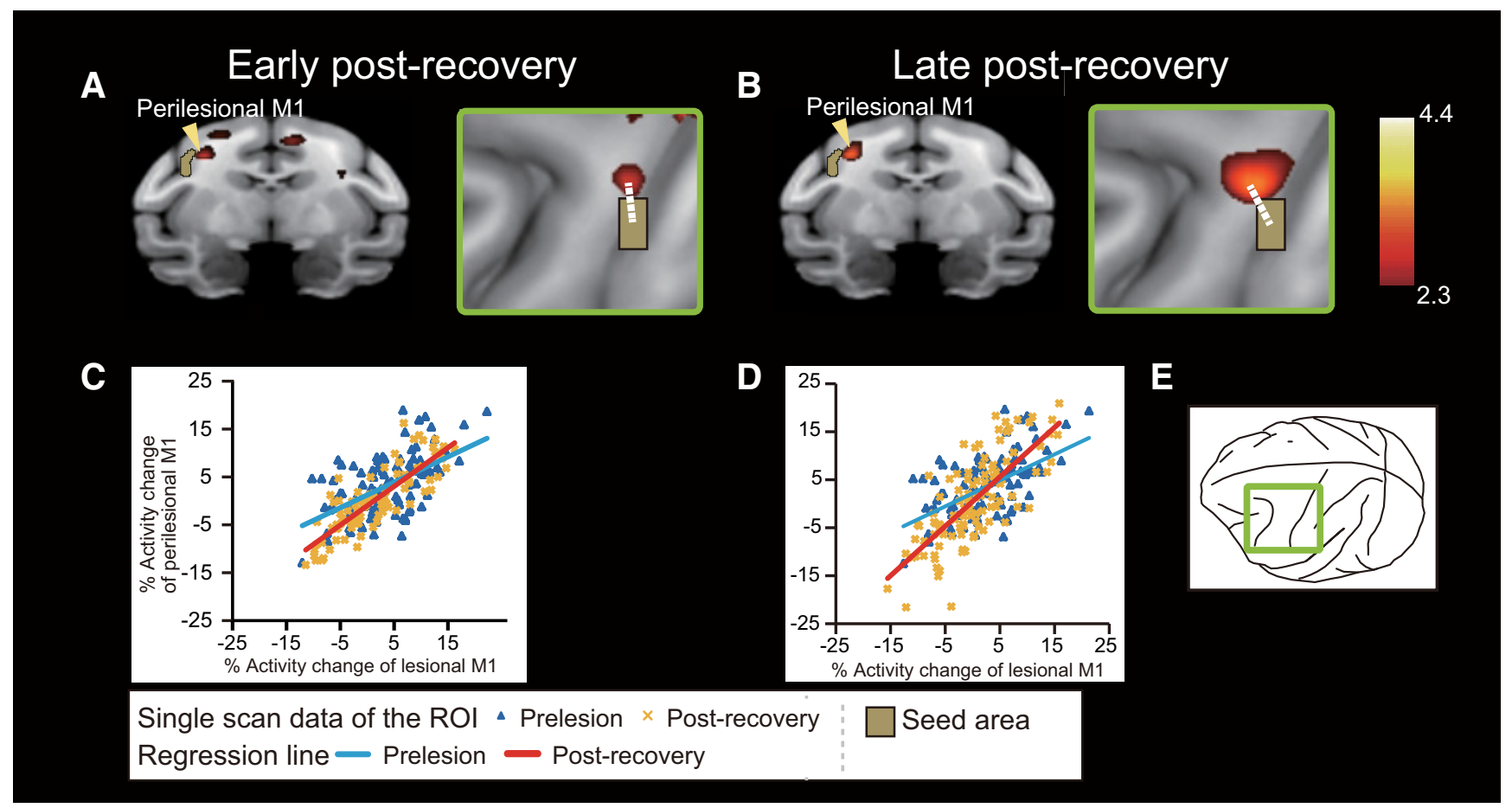

Figure 4. PPI analysis revealed increased functional connectivity within M1 during functional recovery. $A, B$, During recovery, there was a change in the functional correlation between activity in the lesion area and activity in the perilesional $\mathrm{M} 1$ (arrowheads). Areas in which activation changed ( $p<0.01$ uncorrected, $Z>2.3$ ) are superimposed on a 3D reconstruction of a macaque monkey brain MRI template. The brown region represents the seed region, which includes the lesion area in M1. Right panels, PPI effects around the central sulcus from a view perpendicular to the cortical surface, as shown in $\boldsymbol{E}$. White dotted lines indicate the distance between the center of the lesion area and the peak voxel in the rostromedial M1 (early postrecovery, $3.6 \mathrm{~mm}[\boldsymbol{A}]$; late postrecovery, $3.8 \mathrm{~mm}[\boldsymbol{B}])$. The significance level is given in terms of a $Z$ score represented on a color scale. $\boldsymbol{C}, \boldsymbol{D}$, Graphs show representative changes in interactions between activity in the lesioned area and activity in the perilesional M1 during the precision grip task in the early $(\boldsymbol{C})$ and late $(\boldsymbol{D})$ postrecovery periods. Blue triangles and blue regression lines represent prelesion data. Yellow crosses and red regression lines represent data during the postrecovery periods.

Table 3. The cluster coordination of the PPI analysis during the early and late postrecovery periods ${ }^{a}$

\begin{tabular}{|c|c|c|c|c|c|}
\hline Area & Side & Zscore & $x$ & $y$ & $z(\mathrm{~mm}$ \\
\hline \multicolumn{6}{|c|}{ Early postrecovery (precision grip - control) $>$ prelesion (precision grip - control) } \\
\hline M1 & Ipsi & 2.98 & 15 & 16 & 28 \\
\hline S1 & Ipsi & 4.43 & 0 & 8 & 30.5 \\
\hline PMd & Ipsi & 2.92 & 6.5 & 27 & 31.5 \\
\hline Area 8 & Ipsi & 2.87 & 10.5 & 35 & 25 \\
\hline $\mathrm{Cb}$ & Contra & 3.10 & -2.5 & -4.5 & 25 \\
\hline \multicolumn{6}{|c|}{ Late postrecovery (precision grip - control) $>$ prelesion (precision grip - control) } \\
\hline M1 & Ipsi & 3.50 & 16 & 16 & 28 \\
\hline S1 & Ipsi & 3.33 & 1 & 6 & 32 \\
\hline Put & Ipsi & 2.82 & 9 & 24 & 18 \\
\hline Amg & Ipsi & 2.86 & 6 & 23 & 3 \\
\hline 7op & Ipsi & 3.03 & 185 & 22 & \\
\hline $\mathrm{Cb}$ & Ipsi & 3.59 & 3 & -14 & 12 \\
\hline V1 & Ipsi & 2.84 & 5 & -29 & 10 \\
\hline $\mathrm{Cb}$ & Contra & 3.54 & -5 & -6 & 9 \\
\hline
\end{tabular}

${ }^{a} Z$ scores of the peak voxels are indicated. The locations are indicated by the positions along the $x$ (ipsilateralcontralateral), $y$ (rostrocaudal), and $z$ (dorsoventral) axes of the Horsley-Clarke stereotaxic coordinates. Amg, Amygdala; Cb, cerebellar cortex; contra, contralesional hemisphere; ipsi, ipsilesional hemisphere; PMd, dorsal premotor area; Put, putamen; $\mathrm{S1}$, primary somatosensory area; $\mathrm{V1}$, area $\mathrm{V1} ; 70 \mathrm{p}$, parietal operculum.

tion site for M1, in which ICMS elicited digit movements with the lowest threshold. Multiple injections were performed for PMv-h, in which 1.5 $\mu \mathrm{l}$ of muscimol solution was injected into four sites ( 2 tracks $\times 2$ depths, spaced at $3 \mathrm{~mm}$ intervals and centered on the locus with the lowest ICMS threshold for digit movements among PMv). The depth of the injection was determined as the point where the movement threshold of ICMS was the lowest. Multiple injections were also performed for the PMv rostrolateral to the hand area (PMv-rl), the lateral bank of the lower arcuate sulcus, and the adjacent convexity region $3 \mathrm{~mm}$ rostral to PMv-h.
The effects of muscimol injection on hand performance were evaluated by using a small-object retrieval task (precision grip task) as described above. The test session to evaluate hand performance, which consisted of 20 trials, was conducted 30,60, and 120 min after the muscimol injection. Only the results of the test at $120 \mathrm{~min}$ after the injection are shown in Figures 5 and $6 B, C$ because the effect was largest at this time point. The success rate of the precision grip in the task was evaluated by using video analysis. A successful precision grip was defined as a grip in which the tip of the index finger and thumb were both used to retrieve the object, whereas alternate grips were those in which the dorsal part of the index finger, thumb, or middle finger was used. Another type of alternate grip was also observed in which the monkeys raked the object out of the slit by using a single digit. A blind procedure was used to evaluate the success rate of the precision grip; captured video images of monkeys retrieving the object were judged as a success or failure by viewing the images with a lack of knowledge regarding the experimental conditions. The inactivation study for each injection site was performed with an interval of $>2 \mathrm{~d}$ because the effects of muscimol may persist until the day after the injection. Despite this precaution, slight deficits in hand movements were observed before the injection in 7 of the 30 muscimol injection experiments, and behavioral performance levels were below the $95 \%$ confidence level in each recovery period. This may be because the effect of muscimol remained for $>2 \mathrm{~d}$. Even when the level of performance before muscimol injection is low, the areas affected by the drug are thought to be causally related to behavioral performance if it is significantly impaired by a muscimol injection. However, the effects of muscimol inactivation may be underestimated when a muscimol injection results in a slight but statistically nonsignificant deficit in behavioral performance. The result of Mk-Ki in ipsilesional PMv-h during the late postrecovery period (see Fig. $5 B$ ) is consistent with this latter case, suggesting that statistically significant effects of the muscimol injection might have been overlooked in this experiment under the present experimental conditions. Because of the possible lingering effect of muscimol, 
A

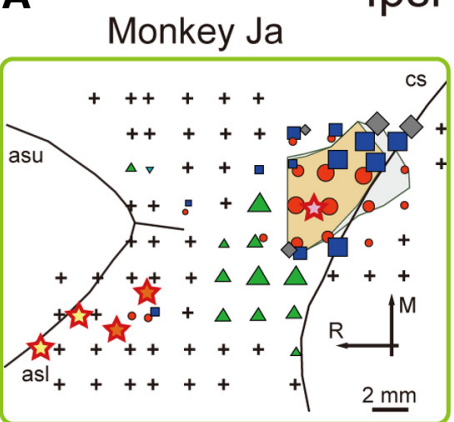

B

(\% Success rate)
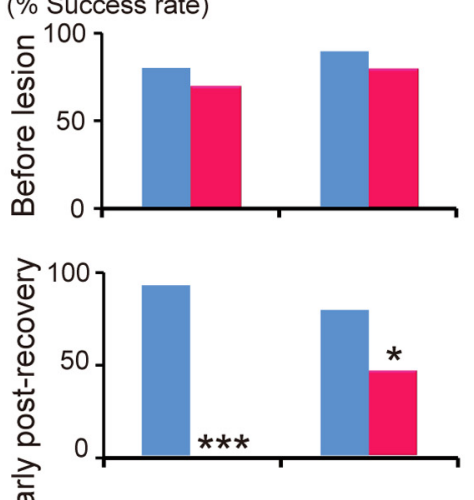

흐

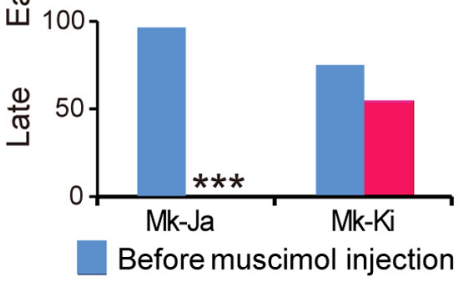

Ipsi-lesion
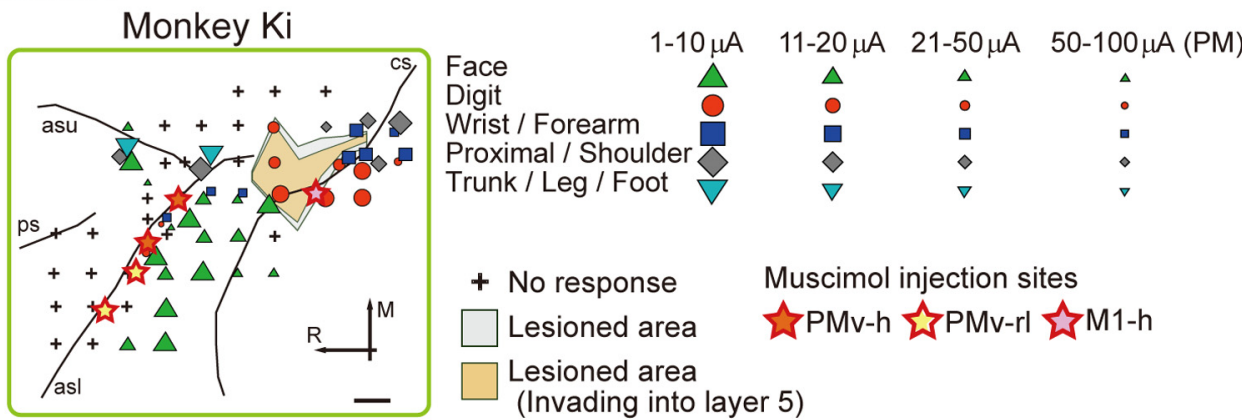

+ No response

Muscimol injection sites

Lesioned area

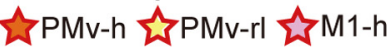

Lesioned area

(Invading into layer 5)
C
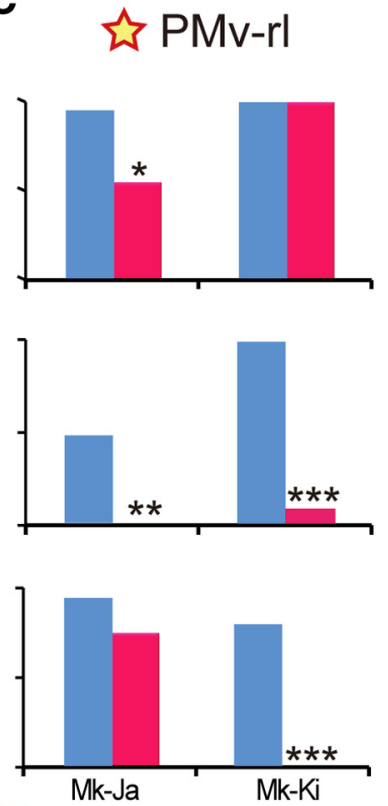

Mk-Ja
D
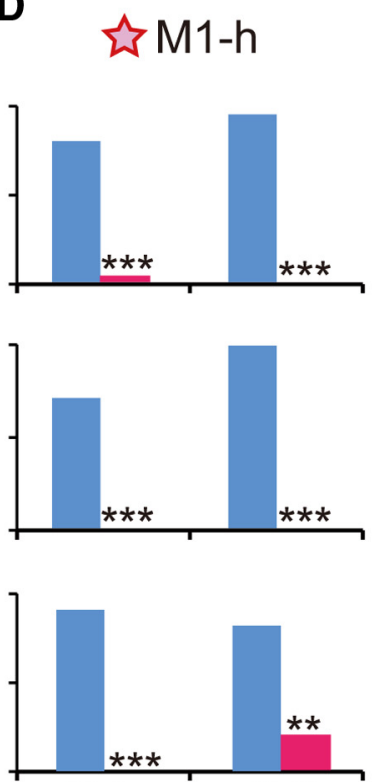

Mk-Ja
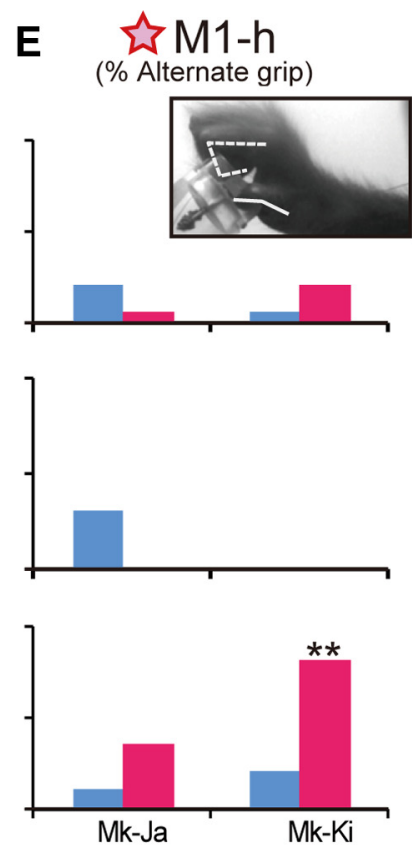

$2 \mathrm{hr}$ after muscimol injection
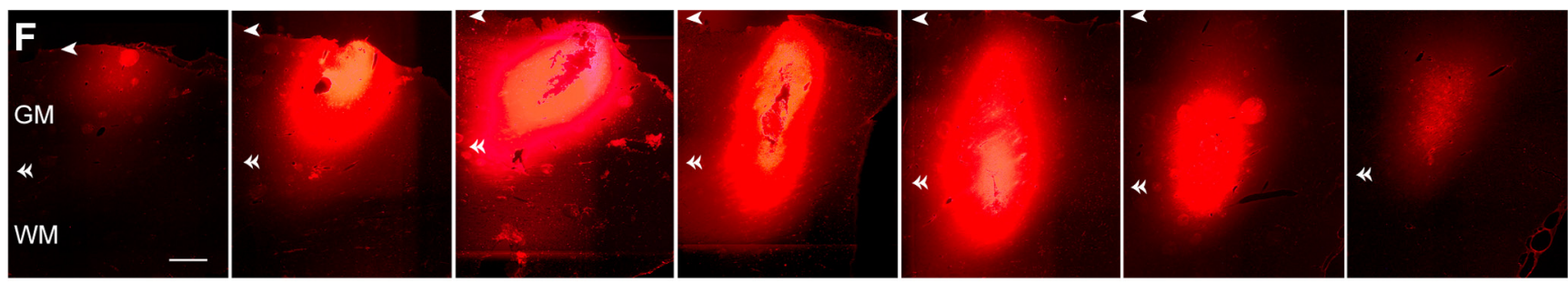

Figure 5. Effects of inactivating the PMv and $M 1$ of the ipsilesional hemisphere on hand movements. $A$, The topographic motor representations of PMv and $M 1$ derived before lesion, from a view perpendicular to the cortical surface. Movements elicited at the threshold of ICMS are indicated by the symbols. Some electrode penetration sites showed no response to ICMS at current strengths up to either $50 \mu \mathrm{A}$ for M1 or $100 \mu \mathrm{A}$ for PMv. Gray and brown areas represent areas of histologically confirmed lesions. Colored stars represent sites of muscimol injection. Arrows indicate rostral-caudal and medial-lateral orientations. R, Rostral; M, medial; asl, lower arcuate sulcus; asu, upper arcuate sulcus; CS, central sulcus. B-D, The effects of inactivating PMv, in which ICMS elicited movements of hand digits (PMv-h, $B$ ), the PMv area rostrolateral to PMv-h (PMv-rl, $C$, or the hand digit area of $M 1(M 1-h, D)$. The success rates of precision grip in two monkeys before the M1 lesion and during the early and late postrecovery periods after the lesion are shown. Muscimol injections into both the ipsilesional PMv-h and PMv-rl in the early postrecovery period resulted in a significant deficit in performing the precision grip in both monkeys. ${ }^{*} p<0.01$ (Fisher's exacttest). ${ }^{* *} p<0.001$ (Fisher's exact test). ${ }^{* * *} p<0.0001$ (Fisher's exact test). In the late postrecovery period, muscimol injection into either of the areas (Monkey [Mk]-Ja, PMv-h; Mk-Ki, PMv-rl) resulted in a deficit of the precision grip. E, The effects of inactivating the ipsilesional M1-hon the rate of use of an alternate grip (i.e., types of grasping other than precision grip). Inactivating the primary motor cortex (M1-h) during the late postrecovery period resulted in the impairment of movements that depend heavily on distal digits, such as the precision grip, whereas proximal movements remained intact. Muscimol injection into the ipsilesional M1-h during the late postrecovery period impaired precision grip but, in contrast to that before lesion, did not impair whole-hand grip in either monkey. Consequently, the monkeys retrieved the object by using an alternate grip, in which the object was held between the thumb and around the proximal joint of the index finger. Inset photograph, An example of the alternate grip in Mk-Ki. $\boldsymbol{F}$, Serial coronal sections of M1 spaced by $0.8 \mathrm{~mm}$ showing the extent of diffusion of $3.0 \mu l$ fluorescent muscimol. Arrowheads and double arrowheads indicate the locations of the cortical surface and the boundaries between the gray and white matter, respectively. GM, Gray matter; WM, white matter. Scale bars: $A, 2 \mathrm{~mm} ; \boldsymbol{F}, 1 \mathrm{~mm}$.

we decided not to perform both PET imaging and muscimol injection studies in the same monkeys. Injection of saline into each muscimol injection site, both before and after the M1 lesion, did not result in any deficit of hand movements (data not shown).
Fluorescent muscimol (BODIPY TMR-X-conjugated muscimol, Invitrogen) was used to determine the extent of the spread of muscimol. The area affected by an injection of muscimol can only be verified conclusively by directly monitoring neuronal activity during the period of 


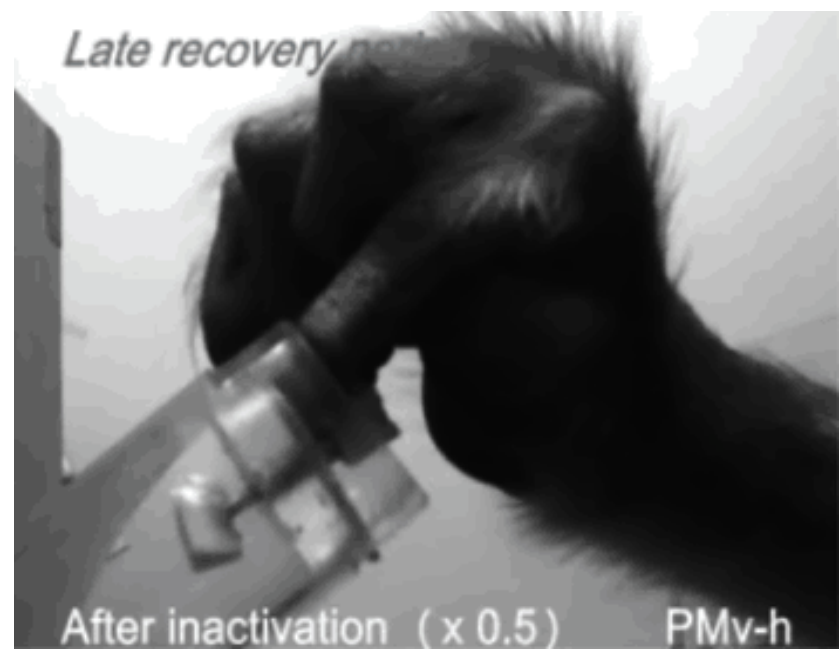

Movie 1. The effects of inactivating an area of the ventral premotor cortex in the ipsilesional hemisphere, in which ICMS elicited movements of hand digits (PMv-h), on performance in the precision grip task.

inactivation within the same animal. However, the technical hurdles involved in such an experiment are extremely high. Therefore, as the next best option, we estimated the extent of inactivation due to an injection of muscimol by evaluating the spread of fluorescent muscimol injected in a separate monkey. The same concentration $(5 \mu \mathrm{g} / \mu \mathrm{l}$, dissolved in $0.1 \mathrm{M}$ phosphate buffer at $\mathrm{pH} 7.4)$ and volumes $(1.5$ or $3.0 \mu \mathrm{l})$ as used in the behavioral experiments were injected into the M1 of another monkey. The monkey was perfused as described above, and the extent of diffusion was assessed in coronal sections (see Fig. $5 F$ ) by using a fluorescence microscope (BZ-8100, Keyence).

Statistical analyses. Statistical significance was assessed by Fisher's exact test (see Figs. 5 and 6), a nonparametric test. All of the tests were performed two-sided.

\section{Results}

\section{Behavioral results}

Before the lesion, all of the monkeys smoothly performed the precision grip task shown in Figure $1 A$. Ibotenic acid injection in the M1 hand digit area resulted in a focal lesion in which there was loss of neurons and gliosis (Fig. 1C,D); the average percentage of lesion volume for the whole M1 digit area was $74.5 \pm 7.6 \%$ (Table 1). Immediately afterward, a lesion of the hand digit area in $\mathrm{M} 1$ (Fig. $1 C-E$ ) was made by ibotenic acid injection, the monkeys could not perform a small-object retrieval task because of hand paralysis, including a complete loss of digit movement (Fig. $1 A)$. Functional recovery of hand movements was observed during the course of daily postlesion training on the precision grip task. Specifically, the number of successful trials in the precision grip task progressively increased during the first month after the lesion (Fig. 1A,B).

\section{PET imaging study}

We performed a PET brain imaging study to reveal the changes in brain activity that potentially underlie recovery of the precision grip. By scanning the animals' brains by using $\mathrm{H}_{2}{ }^{15} \mathrm{O}$-PET while they performed the retrieval task, we evaluated the regional cerebral activation that was related to performance in the precision grip task in the same animals at three stages: prelesion, early postrecovery, and late postrecovery periods. Before the lesion, task-related increases in $\mathrm{rCBF}$ were found in the putative hand area of M1 contralateral to the used hand and in other areas, including the contralateral anterior intraparietal area and ipsilateral cerebellum (Fig. 2A; Table 2).

PET scans after recovery of precision grip confirmed that taskrelated activation in the putative $\mathrm{M} 1$ hand area was reduced by the lesion (Fig. 3), whereas other cortical regions, such as the PMv, showed enhanced activation (Figs. $2 B$ and 3; Table 2). Indeed, comparison of task-related activity at the early postrecovery period (1-2 months after the M1 lesion) with that observed before the lesion revealed that such activity increased in clusters located in the bilateral PMv (Figs. $2 B$ and 3). The clusters in both the ipsilateral and contralesional PMv were extended along the lower arcuate sulcus (for the location of the sulcus, see Fig. 5A), being located at the lateral bank of the lower arcuate sulcus and the adjacent convexity region (Fig. $2 B$, double arrowheads). Increased task-related activity at this stage was also found in other brain areas, including the secondary somatosensory area (S2) of the contralesional hemisphere and the bilateral cerebellum (Table 2). Comparison of task-related activity in the late postrecovery period (3-4 months after the M1 lesion) with that before the lesion revealed a prominent increase in the activity of the cluster in the ipsilesional but not contralesional PMv (Figs. $2 \mathrm{C}$ and 3; Table 2).

Another possible compensatory change in the perilesional M1 was suggested from the results of PPI analysis (Fig. 4). The peak of the PPI effect was located rostromedial to the lesion area; the distance between the peak and the center of the lesion was $3.6 \mathrm{~mm}$ and $3.8 \mathrm{~mm}$ for the early and late postrecovery periods, respectively (Fig. $4 A, B$, arrows). The PPI effect in the perilesional M1 was more prominent during the late postrecovery period than during the early postrecovery period (Fig. 4; Table 3). The prominent PPI effects during the late postrecovery period were also observed in the ipsilesional putamen and contralesional cerebellum, which may function in coordination with the perilesional M1 (Table 3).

\section{Pharmacological inactivation study}

To confirm whether the changes in brain activity in the PMv and perilesional M1 shown by the PET imaging study are causally involved in the recovery of manual dexterity, we performed another experiment using pharmacological inactivation. If a causal relationship exists, inactivation of these areas should result in the loss of recovered motor function. To test this conjecture, we performed focal and transient inactivation of a target cortical region by microinjecting muscimol, a $\mathrm{GABA}_{\mathrm{A}}$ receptor agonist.

$\mathrm{PMv}-\mathrm{h}$ was defined as the cortical region where ICMS elicited hand movements. The effects of muscimol injection $(5 \mu \mathrm{g} / \mu \mathrm{l}, 4$ injection sites, $1.5 \mu \mathrm{l}$ each) in this region before and after the M1 lesion were compared. Moreover, we investigated the effects of muscimol injection into the PMv-rl (Fig. 5A), in which ICMS elicited no movement, because involvement of this area, including the lateral bank of the lower arcuate sulcus and the adjacent convexity region, was suggested by the results of PET imaging (Fig. 2B). Inactivation of PMv-h had a small but insignificant effect on precision grip before the M1 lesion (Fig. $5 B$ ). In contrast, inactivation of PMv-rl impaired precision grip in one of the two monkeys examined, suggesting that there are individual differences in the contribution of this area to precision grip in intact monkeys (Fig. 5C).

During the early postrecovery period, inactivation of either $\mathrm{PMv}-\mathrm{h}$ or PMv-rl of the ipsilesional hemisphere significantly impaired precision grip in both monkeys examined (Fig. $5 B, C$; Movie 1; Fisher's exact test, $p<0.01$ ), suggesting that both of these areas are involved in functional recovery of the precision 
A

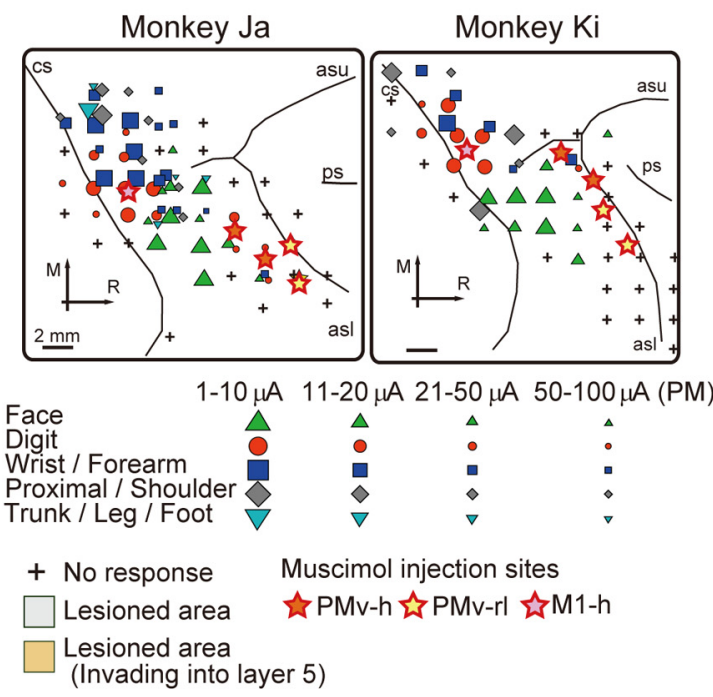

B

(\% Success rate)
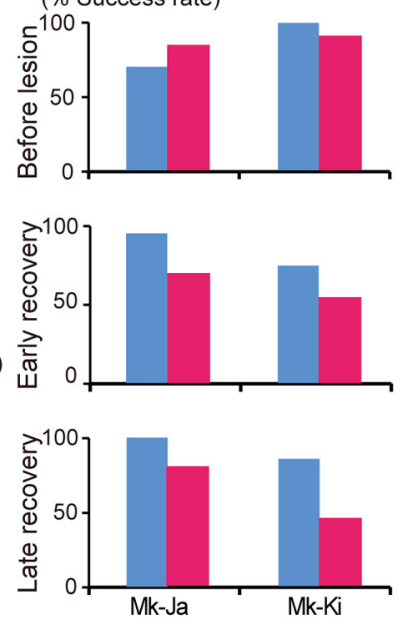

Before muscimol injection
C $\lesssim \mathrm{PMv}-\mathrm{rl}$
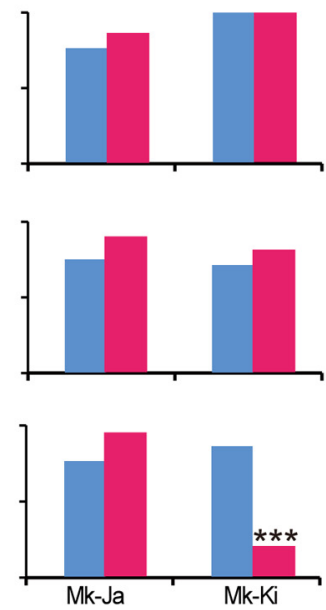

$2 \mathrm{hr}$ after muscimol injection

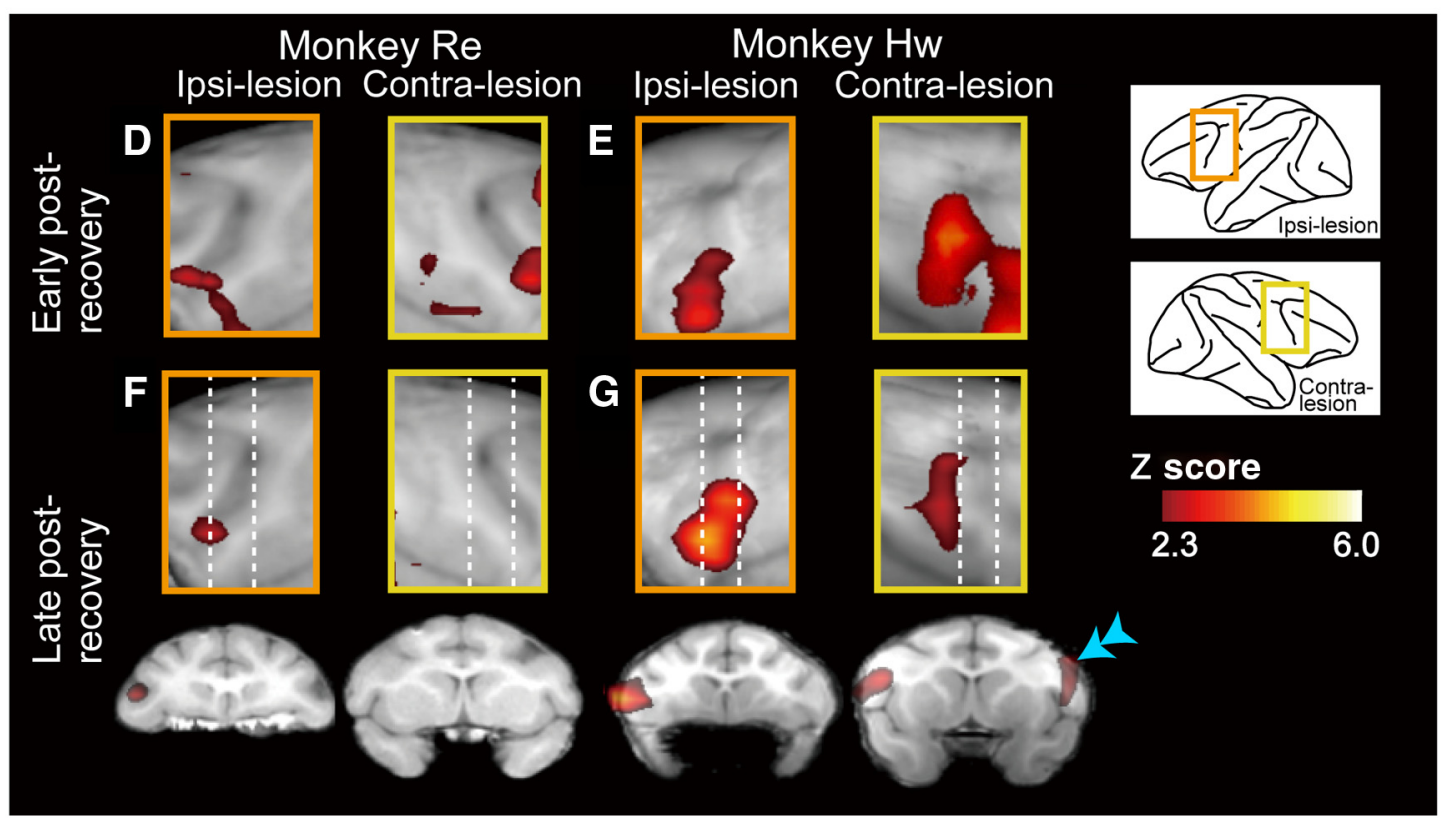

Figure 6. Effects of inactivating the PMv of the contralesional hemisphere on the performance of precision grip. $A$, Topographic motor representation of the PMv and M1 of the contralesional hemisphere derived before lesion. Symbols and abbreviations are the same as in Figure $5 \boldsymbol{A} . \boldsymbol{B}, \boldsymbol{C}$, Effects of inactivating PMv, in which ICMS elicited movements of hand digits (PMv-h, $\boldsymbol{B}$ ), or the PMv area rostrolateral to PMv-h (PMv-rl, C), on the performance of precision grip. Muscimol injections into the contralesional PMv-rl in the late postrecovery period resulted in a significant deficit in performing the precision grip in Mk-Ki. ${ }^{* * *} p<0.0001$ (Fisher's exact test). $\boldsymbol{D}, \boldsymbol{E}$, Activation during the early postrecovery period (rCBF during the precision grip task; rCBF during the control task) was compared with that during the prelesion stage in each monkey: $\mathrm{Mk}-\mathrm{Re}(\boldsymbol{D})$ and $\mathrm{Mk}-\mathrm{Hw}(\boldsymbol{E})$. Brain areas with significantly increased $\mathrm{rCBF}$ are superimposed on a lateral view of the frontal lobe. $\boldsymbol{F}, \mathbf{G}$, Activation during the late postrecovery period was compared with that during the prelesion stage in each monkey: Mk-Re $(\boldsymbol{F})$ and $\mathrm{Mk}$-Hw $(\boldsymbol{G})$. Bottom row, Coronal sections during the late postrecovery period at the level of the dotted lines. During the late postrecovery period, increased activation of the contralesional PMv was observed in Mk-Hw (double arrowhead) but not in Mk-Re. Significance level is given in terms of a $Z$ score represented on a color scale.

grip. In contrast, smaller subregions of PMv are involved in functional recovery during the late postrecovery period; inactivation of the ipsilesional PMv-h impaired precision grip in one monkey and inactivation of the ipsilesional PMv-rl impaired precision grip in the other (Fig. 5 B, C; Fisher's exact test, $p<0.0001$ ). These results are consistent with those obtained from the PET imaging study, in which increased activity was observed in a relatively large area of the ipsilesional PMv during the early postrecovery period, whereas activation of the ipsilesional PMv during the late postrecovery period was restricted to a more local region (Fig. $2 B, C$, double arrowheads).
Although increased activity in the contralesional PMv was shown by PET imaging during the early postrecovery period (Fig. $2 B$ ), neither inactivation of the contralesional PMv-h nor that of the contralesional PMv-rl impaired precision grip during the early postrecovery period (Fig. $6 B, C$ ). Inactivation of the contralesional PMv-h during the late postrecovery period also had no effect on precision grip in either monkey. Individual differences were observed for inactivation of the contralesional PMv-rl during the late postrecovery period; the treatment affected precision grip in one monkey (Mk-Ki, Fig. $6 C$; Fisher's exact test, $p<$ $0.0001)$ but not the other. Although the reason for this difference 


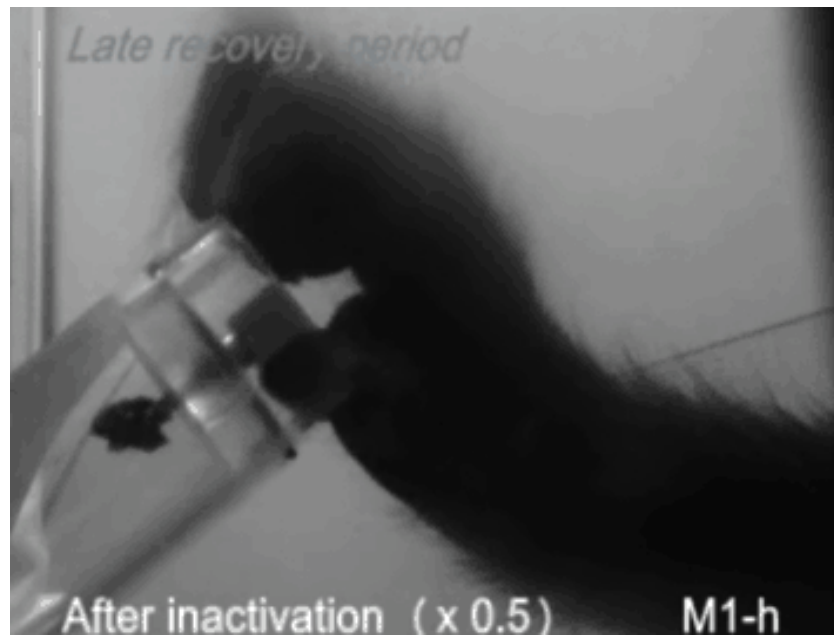

Movie 2. The effects of inactivating the hand area of the primary motor cortex (M1-h) of the ipsilesional hemisphere on performance in the precision grip task. The monkey retrieved the object by using an alternate grip other than the precision grip after inactivation during the late recovery stage.

is unclear, the PET imaging results also indicated individual differences in the activation of the contralesional PMv during the late postrecovery period; increased activation of the contralesional PMv was observed in one monkey but not the other (Fig. $6 F, G)$. Such intersubject variability was less prominent during the early postrecovery period (Fig. $6 D, E$ ). The contribution of the contralesional PMv to functional recovery during the late postrecovery period may vary because of slight differences in the location and size of the lesion, the behavioral environment after the lesion, or both.

The PPI analysis of PET data for functional connectivity also suggests a compensatory change in brain activity in the perilesional M1. Therefore, we also conducted an inactivation study in M1. Inactivation of M1-h during the early postrecovery period resulted in the impairment of hand movements, as was observed by inactivation before the M1 lesion (Fig. 5D, E; Movie 2). Inactivation before the lesion and during the early postrecovery period impaired hand movements, including both whole-hand and precision grips. The spread of fluorescent muscimol in monkey M1 suggested that a $1.5 \mu \mathrm{l}$ injection of muscimol inactivated a spherical volume of brain tissue $\sim 3-4 \mathrm{~mm}$ in diameter, and a 3.0 $\mu \mathrm{l}$ injection of muscimol inactivated a spherical volume of brain tissue $\sim 5-6 \mathrm{~mm}$ in diameter (Fig. $5 F$ ). The present result for the $1.5 \mu \mathrm{l}$ injection is consistent with the results of previous studies, which showed that a $1 \mu$ l injection of muscimol inactivated a spherical volume of brain tissue $\sim 3-4 \mathrm{~mm}$ in diameter (Partsalis et al., 1995; Arikan et al., 2002). Therefore, the $3.0 \mu \mathrm{l}$ of muscimol injected in M1-h after the M1 lesion should spread beyond the lesional area and inactivate the perilesional area, in which topographic maps of both the undamaged hand digit area and areas for other body parts, such as the wrist, shoulder, and face, are included (Fig. 5A). The observed spread of fluorescent muscimol suggests that the injection muscimol into M1 did not inactivate the PMv and that the injections of muscimol into PMv-h or $\mathrm{PMv}$-rl did not inactivate the perilesional M1. It is possible, however, that the injection of muscimol into the PMv-h of Mk-Ki may have inactivated the face area of M1 because these two subregions were adjacently located in this monkey (Fig. $5 A$ ).

Muscimol injection into the ipsilesional M1-h during the late postrecovery period also impaired precision grip. In contrast to that before the lesion and during the early postrecovery period, however, inactivation during the late postrecovery period did not impair whole-hand grip in either monkey, and the monkeys retrieved the small object by using an alternate grip other than the precision grip (Fig. 5E; Movie 2). Therefore, muscimol injection into the M1-h during the late postrecovery period resulted in the impairment of movements that depend heavily on distal digits, such as precision grip while proximal movements remained intact. The area inactivated by the injection probably includes the area rostromedial to the lesion, which is where the peak of the PPI effect was located in the PET imaging (Fig. $4 B$, white dotted line; the distance from the center of the lesion to the peak of the PPI effect was $3.8 \mathrm{~mm}$, whereas that to the edge of a significant PPI effect was $1.6 \mathrm{~mm}$ ). Therefore, the results from the PET imaging and pharmacological inactivation studies suggest that functional reorganization of the perilesional area occurred such that the area serving proximal movements came to be involved in distal movements, including precision grip.

Inactivation of contralesional M1 ( $3 \mu \mathrm{l}$ in a single site) during both the early and late postrecovery periods did not significantly affect performance of the precision grip $(p>0.01$; data not shown).

\section{Discussion}

In the present study, we showed that, following lesioning of the M1 digit area, functional reorganization occurs in the remaining undamaged motor cortex in a time-dependent manner. Among the areas of the remaining undamaged motor cortex, the ipsilesional PMv was shown by both functional brain imaging and inactivation analyses to contribute to the recovery of dexterous hand movements. The present finding is consistent with that of a previous study in squirrel monkeys, which showed changes in the topographic motor representation of PMv after a focal lesion in the hand digit area of M1 (Frost et al., 2003). The present study further demonstrates functional reorganization associated with the performance of dexterous hand movements in behaving monkeys. The involvement of dorsal premotor cortex in the recovery of hand movements has also been reported in other studies with M1-lesioned monkeys and human stroke patients, both of which showed relatively larger brain damage and incomplete recovery of dexterous hand movements (Liu and Rouiller, 1999; Johansen-Berg et al., 2002; Fridman et al., 2004). The present PET imaging study, however, did not show prominent changes in this area, although it did indicate that PMv is more involved in the recovery of hand movements than is dorsal premotor cortex, at least when the lesion is confined to the hand digit area of M1. This result is consistent with the notion that $\mathrm{PMv}$ is the core constituent of the parietofrontal circuit that underlies dexterous hand movements (Fagg and Arbib, 1998; Rizzolatti and Luppino, 2001).

The involvement of PMv during recovery after a lesion of the cervical spinal cord was also indicated by our previous studies (Nishimura et al., 2007; Higo et al., 2009). Although a direct comparison of spinal cord and motor cortex lesions is difficult, common mechanisms for functional recovery may exist because both lesions affect corticomotoneuronal connections that arise from $\mathrm{M} 1$, which are thought to be essential for manual dexterity. We note that a number of differences in functional recovery mechanisms may also exist between spinal cord and motor cortex lesions. For example, whereas involvement of the contralesional M1 during functional recovery after a spinal cord lesion was indicated, the results of the present study indicate no such involve- 
ment of the contralesional M1 in the functional recovery after lesions of M1.

The present study suggests that in addition to the subarea of the ipsilesional PMv, in which ICMS elicited hand movements (PMv-h), the subarea rostrolateral to PMv-h (PMv-rl) also contributes to functional recovery of the precision grip. PMv-rl includes areas F5a and F5c; these areas contain mirror neurons, a type of neuron that is activated when the monkey performs a particular action, such as grasping an object, while observing a similar action being performed by a peer (Fabbri-Destro and Rizzolatti, 2008). The original function of mirror neurons is presumably to provide visual feedback on the shape of the monkey's own hand during grasping (Oztop and Arbib, 2002). This feedback system may be reinforced during functional recovery of hand movements.

In contrast to the results of the ipsilesional PMv, inactivation of the contralesional PMv, either PMv-h or PMv-rl, did not impair the precision grip during the early postrecovery period; however, enhanced activity of the contralesional PMv was indicated by PET imaging at this stage. Together, these findings suggest that the increased brain activity in the contralesional PMv was not critically involved in the recovery of dexterous hand movements. Although increased activity in the contralesional motor cortex has been reported in several fMRI studies of stroke patients (Johansen-Berg et al., 2002; Ward et al., 2003; Rehme et al., 2011), the present results provide important information that increased activity alone does not always indicate involvement in the recovery of manual dexterity.

In addition to the ipsilesional PMv, changes of activity in the perilesional M1 were also shown by PET imaging and PPI analysis. Moreover, the results of our pharmacological inactivation analysis support the idea that the perilesional M1 plays a role in the recovery of manual dexterity. The present study is the first to confirm the appropriateness of using lesions and PPI analysis to address the effective functional connectivity that underlies the plastic response after a local lesion. Whereas both PET imaging and pharmacological inactivation analyses indicated that changes in the ipsilesional PMv were more prominent during the early rather than late postrecovery period, those in the perilesional M1 were rather more prominent during the late postrecovery period. The plastic response in the ipsilesional PMv was identified by enhanced behavior-related activity, whereas that of the perilesional M1 was identified by increased functional connectivity. These findings suggest that multiple steps (and hence neural mechanisms) underlie the functional reorganization and that these steps vary depending on time after the M1 lesion.

We should note that ibotenic acid is a glutamate receptor agonist that produces selective irreversible damage to neurons while leaving glial cells and cerebral vessels intact. Therefore, the neural sequelae induced by an ibotenic acid lesion may be different from those induced by a stroke, a major cause of brain damage in humans, in which occlusion or hematoma of a cerebral vessel occurs. Nevertheless, the mechanisms that underlie functional recovery are thought to be, at least in part, common between them. Further analyses by using animal stroke models will be important in applying the present findings to the development of novel therapies, technologies, or medications to improve the functional recovery of stroke patients.

One of the characteristics of the primate motor cortex is that it is organized into functionally specialized areas that are hierarchically arranged to execute sophisticated movements, such as dexterous hand movements (Tanji, 2001; Dum and Strick, 2002). Here we revealed that time-dependent plastic changes in both the remaining primary motor and premotor cortical areas occur after M1 lesion and that these plastic changes are partly involved in functional recovery; PMv, an area distant from the core of the lesion, plays an important role during the early postrecovery period, whereas the perilesional M1 contributes to functional recovery especially during the late postrecovery period. The current results extend our understanding of the brain plasticity that underlies rehabilitative training-induced motor recovery after brain damage.

\section{References}

Arikan R, Blake NM, Erinjeri JP, Woolsey TA, Giraud L, Highstein SM (2002) A method to measure the effective spread of focally injected muscimol into the central nervous system with electrophysiology and light microscopy. J Neurosci Methods 118:51-57. CrossRef Medline

Courtine G, Bunge MB, Fawcett JW, Grossman RG, Kaas JH, Lemon R, Maier I, Martin J, Nudo RJ, Ramon-Cueto A, Rouiller EM, Schnell L, Wannier T, Schwab ME, Edgerton VR (2007) Can experiments in nonhuman primates expedite the translation of treatments for spinal cord injury in humans? Nat Med 13:561-566. CrossRef Medline

Dancause N, Barbay S, Frost SB, Plautz EJ, Chen D, Zoubina EV, Stowe AM, Nudo RJ (2005) Extensive cortical rewiring after brain injury. J Neurosci 25:10167-10179. CrossRef Medline

Dum RP, Strick PL (2002) Motor areas in the frontal lobe of the primate. Physiol Behav 77:677-682. CrossRef Medline

Fabbri-Destro M, Rizzolatti G (2008) Mirror neurons and mirror systems in monkeys and humans. Physiology (Bethesda) 23:171-179. CrossRef Medline

Fagg AH, Arbib MA (1998) Modeling parietal-premotor interactions in primate control of grasping. Neural Netw 11:1277-1303. CrossRef Medline

Fridman EA, Hanakawa T, Chung M, Hummel F, Leiguarda RC, Cohen LG (2004) Reorganization of the human ipsilesional premotor cortex after stroke. Brain 127:747-758. CrossRef Medline

Friel KM, Nudo RJ (1998) Recovery of motor function after focal cortical injury in primates: compensatory movement patterns used during rehabilitative training. Somatosens Mot Res 15:173-189. CrossRef Medline

Frost SB, Barbay S, Friel KM, Plautz EJ, Nudo RJ (2003) Reorganization of remote cortical regions after ischemic brain injury: a potential substrate for stroke recovery. J Neurophysiol 89:3205-3214. CrossRef Medline

Glees P, Cole J (1950) Recovery of skilled motor functions after small repeated lesions of motor cortex in macaque. J Neurophysiol 13:137-148.

Hayashi T, Ko JH, Strafella AP, Dagher A (2013) Dorsolateral prefrontal and orbitofrontal cortex interactions during self-control of cigarette craving. Proc Natl Acad Sci U S A 110:4422-4427. CrossRef Medline

Higo N, Nishimura Y, Murata Y, Oishi T, Yoshino-Saito K, Takahashi M, Tsuboi F, Isa T (2009) Increased expression of the growth-associated protein 43 gene in the sensorimotor cortex of the macaque monkey after lesioning the lateral corticospinal tract. J Comp Neurol 516:493-506. CrossRef Medline

Higo N, Kunori N, Takashima I (2013) Compensatory changes in neuronal firing in the perilesional motor cortex: a single unit recording study in the macaque monkey. Soc Neurosci Abstr 566.6.

Jenkinson M, Bannister P, Brady M, Smith S (2002) Improved optimization for the robust and accurate linear registration and motion correction of brain images. Neuroimage 17:825-841. CrossRef Medline

Johansen-Berg H, Rushworth MF, Bogdanovic MD, Kischka U, Wimalaratna S, Matthews PM (2002) The role of ipsilateral premotor cortex in hand movement after stroke. Proc Natl Acad Sci U S A 99:14518-14523. CrossRef Medline

Lee L, Siebner HR, Rowe JB, Rizzo V, Rothwell JC, Frackowiak RS, Friston KJ (2003) Acute remapping within the motor system induced by lowfrequency repetitive transcranial magnetic stimulation. J Neurosci 23: 5308-5318. Medline

Liu Y, Rouiller EM (1999) Mechanisms of recovery of dexterity following unilateral lesion of the sensorimotor cortex in adult monkeys. Exp Brain Res 128:149-159. CrossRef Medline

Mayhew TM (1992) A review of recent advances in stereology for quantifying neural structure. J Neurocytol 21:313-328. CrossRef Medline

Murata Y, Higo N, Oishi T, Yamashita A, Matsuda K, Hayashi M, Yamane S (2008) Effects of motor training on the recovery of manual dexterity after 
primary motor cortex lesion in macaque monkeys. J Neurophysiol 99: 773-786. CrossRef Medline

Nishimura Y, Onoe H, Morichika Y, Perfiliev S, Tsukada H, Isa T (2007) Time-dependent central compensatory mechanisms of finger dexterity after spinal cord injury. Science 318:1150-1155. CrossRef Medline

Nudo RJ, Wise BM, SiFuentes F, Milliken GW (1996) Neural substrates for the effects of rehabilitative training on motor recovery after ischemic infarct. Science 272:1791-1794. CrossRef Medline

O’Shea J, Johansen-Berg H, Trief D, Göbel S, Rushworth MF (2007) Functionally specific reorganization in human premotor cortex. Neuron 54: 479-490. CrossRef Medline

Oztop E, Arbib MA (2002) Schema design and implementation of the grasp-related mirror neuron system. Biol Cybern 87:116-140. CrossRef Medline

Partsalis AM, Zhang Y, Highstein SM (1995) Dorsal Y group in the squirrel monkey: II. Contribution of the cerebellar flocculus to neuronal responses in normal and adapted animals. J Neurophysiol 73:632-650. Medline

Passingham RE, Perry VH, Wilkinson F (1983) The long-term effects of removal of sensorimotor cortex in infant and adult rhesus monkeys. Brain 106:675-705. CrossRef Medline

Rehme AK, Fink GR, von Cramon DY, Grefkes C (2011) The role of the contralesional motor cortex for motor recovery in the early days after stroke assessed with longitudinal FMRI. Cereb Cortex 21:756-768. CrossRef Medline

Rizzolatti G, Luppino G (2001) The cortical motor system. Neuron 31:889901. CrossRef Medline
Smith SM (2002) Fast robust automated brain extraction. Hum Brain Mapp 17:143-155. CrossRef Medline

Sugiyama Y, Higo N, Yoshino-Saito K, Murata Y, Nishimura Y, Oishi T, Isa T (2013) Effects of early versus late rehabilitative training on manual dexterity after corticospinal tract lesion in macaque monkeys. J Neurophysiol 109:2853-2865. CrossRef Medline

Tanji J (2001) Sequential organization of multiple movements: involvement of cortical motor areas. Annu Rev Neurosci 24:631-651. CrossRef Medline

Taub E, Uswatte G, Elbert T (2002) New treatments in neurorehabilitation founded on basic research. Nat Rev Neurosci 3:228-236. CrossRef Medline

Travis AM (1955) Neurological deficiencies after ablation of the precentral motor area in Macaca mulatta. Brain 78:155-173. CrossRef Medline

Vilensky JA, Gilman S (2002) Lesion of the precentral gyrus in nonhuman primates: a pre-medline bibliography. Int J Primatol 23:1319-1333. CrossRef

Ward NS, Brown MM, Thompson AJ, Frackowiak RS (2003) Neural correlates of outcome after stroke: a cross-sectional fMRI study. Brain 126: 1430-1448. CrossRef Medline

Woolrich MW, Ripley BD, Brady M, Smith SM (2001) Temporal autocorrelation in univariate linear modeling of FMRI data. Neuroimage 14: 1370-1386. CrossRef Medline

Worsley KJ, Evans AC, Marrett S, Neelin P (1992) A three-dimensional statistical analysis for CBF activation studies in human brain. J Cereb Blood Flow Metab 12:900-918. CrossRef Medline 